\title{
20. GRAVITATIONAL SORTING AND OVERSTABILITY IN WHITE DWARFS
}

\author{
A. BAGLIN \\ Observatoire de Nice, Nice, France
}

\section{Introduction}

Zones with $\mu$ gradient in stars have been discussed extensively in recent years in relation with the so-called 'semi-convective zone' which forms in massive stars when the convective core shrinks.

When the condition $\nabla_{T} \in\left[\nabla_{T a}, \nabla_{T a}+(\beta / 4-3 \mu) \nabla_{\mu}\right]$ is satisfied $(\beta$ is the classical ratio $\mathrm{P}_{\mathrm{Gas}} / \mathrm{P}_{\text {Total }}, \nabla_{T a}$ and $\nabla_{T}$ the adiabatic and actual temperature gradients, $\nabla \mu$ the molecular weight gradient) motions are locally overstable.

A local treatment of this zone led Kato (1966) to the conclusion that these overstable motions produce an important mixing and that the gradient remains almost adiabatic.

Then Gabriel (1969) and Auré (1970) tried to answer the question whether motions can settle in the whole star taking into account the stabilizing effect of the large radiative regions above and below the semi convective zone. Due to the strong stabilization of the outer radiative zones Auré concluded that no motions can be maintained in the star and then no mixing will be achieved.

The most favorable situation to excite overstability of the whole star would be when the zone of varying molecular weight lies very close to the surface, where the eigenfunction is large. Such a situation could be achieved in white dwarfs. It has been shown by Schatzman (1958) that large gravity can lead to a sorting of the elements and to mild thin zones of varying chemical composition. In the case of a white dwarf with a very thin hydrogen envelope, the zone where hydrogen and helium separate can be very superficial. If the star is not too hot the condition $\nabla_{T a}<\nabla_{T}$ will be satisfied. The purpose of this paper is to examine the influence of this zone on the stability of the star.

\section{The Zone of Sorting of Helium in the Envelopes of White Dwarfs}

We first estimate the extend of the sorting zone and then the speed of sorting. We will assume that the hydrogen-helium transition occurs high in the envelope, the characteristic parameters being:

$$
\begin{aligned}
& \log T=4.12 \\
& \log P=6
\end{aligned}
$$

These parameters have been estimated from hydrogen rich envelope models constructed by Vauclair (1970).

For the sake of simplicity we assume that only fully ionized hydrogen and helium 
are present. Let us call $Z_{1}, n_{1}, m_{1}$, the charge, number density and mass of hydrogen ions; $Z, n, m$ the same quantities for helium ions, $e_{1}, s$ the charge and number density of electrons. We will use the $u$ and $v$ variables defined by:

$$
\begin{aligned}
\mathrm{d} v & =-\frac{m g}{k T} \mathrm{~d} z \\
\frac{\mathrm{d} v}{v}={ }_{k T}^{e \psi^{\prime}} \mathrm{d} z &
\end{aligned}
$$

where $g$ is the acceleration of gravity and $\psi^{\prime}$ the electrical potential.

The statistical equilibrium is:

$$
\begin{aligned}
& n=n_{0} \frac{T_{0}}{T} u^{4} v^{2} \\
& n_{1}=n_{10} \frac{T_{0}}{T} u v \\
& s=s_{0} \frac{T_{0}}{T} u^{m_{e} / m_{1}} v^{-1} \simeq s_{0} \frac{T_{0}}{T} v^{-1}
\end{aligned}
$$

$n_{0}, n_{10}, s_{0} T_{0}$, being the number density of helium, hydrogen and electrons, and the temperature at the same reference level.

The extend of the zone of sorting is given by

$$
\begin{aligned}
& \frac{1 \mathrm{~d} u}{n \mathrm{~d} z}=-\frac{1 \mathrm{~d} T}{T \mathrm{~d} z}+\frac{4 \mathrm{~d} u}{u \mathrm{~d} z}+\frac{2 \mathrm{~d} v}{v \mathrm{~d} z} \\
& \begin{array}{l}
1 \mathrm{~d} u \\
u \mathrm{~d} z
\end{array}=-\begin{array}{ccc}
1 & 1 \mathrm{~d} P \\
R \varrho T \mathrm{~d} z
\end{array}=-\mu \begin{array}{l}
1 \mathrm{~d} P \\
P \mathrm{~d} z
\end{array}
\end{aligned}
$$

The estimate of $1 / v \mathrm{~d} v / \mathrm{d} z$ is obtained using the condition of electrical neutrality which relates $u$ and $v$.

$$
n_{1} Z_{1}+n Z=s
$$

At the reference level one has

$$
n_{10} Z_{1}+n_{0} Z=s_{0}
$$

and at any level

$$
2 \frac{n_{0}}{s_{0}} u^{4} v^{3}+\frac{n_{10}}{s_{0}} u v^{2}=1
$$

If one chooses the reference level as being the level of half sorting

$$
\begin{aligned}
& n_{10}=0.5 \frac{\varrho}{m_{1}} \quad n_{0}=0.5 \frac{\varrho}{4 m_{1}} \\
& \begin{array}{l}
n_{0} \\
s_{0}
\end{array}=\begin{array}{l}
1 \\
6
\end{array} \text { and } \quad \begin{array}{c}
n_{10} \\
s_{0}
\end{array}=\begin{array}{l}
2 \\
3
\end{array}
\end{aligned}
$$


Equation (7) becomes

$$
u^{4} v^{3}+2 u v^{2}-3=0
$$

So that $1 / v \mathrm{~d} v / \mathrm{d} z$ can be obtained by

$$
\frac{1 \mathrm{~d} v}{v \mathrm{~d} z}=-\frac{1 \mathrm{~d} u}{u \mathrm{~d} z}\left(\begin{array}{c}
4 u^{3} v^{3}+2 v^{2} \\
3 u^{3} v^{2}+4 u
\end{array}\right)
$$

In the vicinity of $u=v=1$ this gives

$$
\frac{1 \mathrm{~d} v}{v} \frac{\mathrm{d} z}{2} \simeq-\frac{6}{7} \frac{\mathrm{d} u}{\mathrm{~d} z}
$$

Then Equation (3) is

$$
\frac{1 \mathrm{~d} n_{1}}{n_{1} \mathrm{~d} z}=-\left(\nabla_{T}+\frac{16}{7} \mu\right)_{p}^{1 \mathrm{~d} p}
$$

For a typical white dwarf atmosphere

$$
\left|\begin{array}{ll}
1 \mathrm{~d} u_{1} \\
u_{1} \mathrm{~d} z
\end{array}\right| \frac{1 \mathrm{~d} p}{p \mathrm{~d} z}
$$

So that the extent of the zone of sorting is of the order of half a pressure scale height i.e. 2 temperature scale heights. In the typical case, this leads to a distance of approximately $5 \times 10^{4} \mathrm{~cm}$.

The velocity of the elements, as given by Chapman and Cowling (1960) is

$$
\begin{aligned}
v & =\lambda_{D} k_{1} \frac{1 \mathrm{~d} p}{p \mathrm{~d} z} \\
k_{1} & =2 A-i-1 \\
\lambda_{D} & =6.62 \times 10^{9} T^{5 / 2} /\left(n_{2} M_{2} i \alpha\right)
\end{aligned}
$$

$A, i$, being the molecular weight and the degree of ionisation of the sorted element; $\alpha$ is a slowly varying factor of the order of 10 .

$$
M_{2}=\frac{m}{m_{1}+m}
$$

with the typical values chosen here

$$
V \simeq 10^{-3} \mathrm{~cm} / \mathrm{sec}
$$

So that the sorting is achieved in $5 \times 10^{7} \mathrm{sec}$.

If no mixing processes like turbulence occur in the envelope the sorting is completely achieved during evolution.

The corresponding gradient of molecular weight is of the order of

$$
\nabla \mu=\frac{\mathrm{d} \log \mu}{\mathrm{d} \log p} \simeq 0.1
$$


The extension of the zone $\nabla_{T}<\nabla_{T a}$ in hydrogen rich envelopes of white dwarfs is extremely sensitive to the effective temperature and less sensitive to gravity.

In a typical model $M=0.88 M_{\odot} L=0.003 L_{\odot} \log T_{\text {eff }}=4.12, \log p$ is in the range [5.44;6.59] and $\log T[4.03 ; 4.45]$. Smaller effective temperature leads to strongly convectively unstable models, and larger effective temperature to strongly radiatively stable ones.

\section{Stability of the Gravity Modes}

As the interesting region lies in the very outer layers of the star we assume a plane parallel medium stratified along the $z$ axis. Equations of continuity, motion, radiative equilibrium (in the diffusion approximation) and energy lead to the well known system for the displacement and the perturbations:

$$
\begin{aligned}
& \nabla \cdot r^{\prime}+\frac{\varrho^{\prime}}{\varrho}+z^{\prime} \frac{\partial \log \varrho}{\partial z}=0 \\
& \sigma^{2} r^{\prime}=\stackrel{1}{\varrho} \nabla p^{\prime}+g \stackrel{\varrho^{\prime}}{\varrho} \\
& \nabla\left(\frac{4}{3} a T^{3} T^{\prime}\right)=-{ }_{c}^{\kappa \varrho} F^{\prime}-\left(\begin{array}{c}
\kappa \varrho \\
c
\end{array}\right)^{\prime} F \\
& \left(\begin{array}{l}
p^{\prime} \\
p
\end{array}+z^{\prime} \frac{\partial \log p}{\partial z}\right)-\nabla_{\mathrm{ad}}\left(\begin{array}{l}
\varrho^{\prime} \\
\varrho
\end{array}+z^{\prime} \frac{\partial \log \varrho}{\partial z}\right)=-\frac{\nabla_{\rho a}-1}{i \sigma p}(\nabla \cdot F)^{\prime}
\end{aligned}
$$

$r^{\prime}$ is the displacement and $z^{\prime}$ its component along the $z$ axis; $\varrho, p, T$ density, pressure, temperature; $g$ gravity, along the $z$ axis; $F, \chi$ radiative flux and Rosseland mean opacity.

All perturbed quantities are written in the form

$$
A=A_{0}+A^{\prime}(z) e^{i \sigma t+k_{x} x+k_{y} y}
$$

$A_{0}$ is the equilibrium value and $A^{\prime}$ the eulerian perturbation, function of $z$ only.

We also assume the perfect gas law, with no contribution of the radiative pressure:

$$
P=R \varrho T / \mu
$$

$\mu$ the molecular weight is assumed to be constant along the motion, the diffusion time being much longer than the period of the oscillations; so that

$$
\mu^{\prime} / \mu+z^{\prime} \partial \log \mu / \mathrm{d} z=0
$$

As usual we will carry a linear analysis and the system (15) can be written in terms of the variable $\omega=\log p$ and the reduced functions

$$
\begin{aligned}
& \xi=z^{\prime} / H_{p}, \quad \theta=T^{\prime} / T, \quad \pi=p^{\prime} / p, \quad \varphi=F_{z}^{\prime} / F \\
& \partial \xi=(\nabla \mu-1) \xi+\left(1-\begin{array}{c}
k^{2} g H_{p} \\
\sigma^{2}
\end{array}\right) \pi-\theta
\end{aligned}
$$




$$
\begin{aligned}
& \frac{\partial \pi}{\partial \omega}=\left(\nabla \mu-\frac{\sigma^{2} H_{p}}{g}\right) \xi-\theta \\
& \frac{\partial \theta}{\partial \omega}=\left(1+\kappa_{\mu}\right) \nabla_{\mu} \nabla_{T} \xi+\left(1+\kappa_{p}\right) \nabla_{T} \pi+\left(\chi_{T}-5\right) \nabla_{T} \theta+\nabla_{T} \varphi \\
& \frac{\partial \varphi}{\partial \omega}=-\frac{i \sigma p H_{p}}{F \nabla_{T a}}\left[\left(\nabla_{T}-\nabla_{T a}\right) \xi+\left(1+\nabla \varrho_{a}\right) \pi-\theta\right]+{ }^{2} H_{p}^{2} \theta \\
& \nabla_{T}
\end{aligned}
$$

In atmospheres of white dwarfs the three quantities $k^{2} g H / \sigma^{2}, \sigma^{2} H_{p} / g$ and $k^{2} H_{p}^{2} / \nabla_{T}$ are very small and will be neglected all along.

Auré (1970) has shown that due to the exponential increase of the adiabatic eigenfunction close to the surface the influence of the radiative layers situated below the region of varying molecular weight is negligeable. But a large damping comes from the outer layers. We will assume here that the region of sorting coincides with the photosphere. This means that the star is composed almost entirely of helium and keeps only a thin atmosphere of hydrogen.

To be able to proceed further in the analytical calculations we will make two drastic approximations. In the region of varying molecular weight extending from $z_{1}$ to $z_{0}$ motions are adiabatic, although in the outer layers from $z_{0}$ to $z_{2}$ they are isothermal.

The actual eigenfunction is $\xi=\xi_{0} e^{i \sigma t+\sigma^{\prime t}}$ and the damping coefficient is expressed by (Ledoux and Walraven, 1958)

Let us call

$$
\sigma^{\prime}=\frac{\int_{T}^{\delta T}(\nabla \cdot F)^{\prime} \mathrm{d} v}{\int \varrho r^{\prime} \cdot r^{\prime} \mathrm{d} v}
$$

$$
I_{1}=\int_{z_{1}}^{z_{0}} \frac{\delta T}{T} \nabla \cdot F^{\prime} \mathrm{d} v \text { and } I_{2}=\int_{z_{0}}^{z_{2}} \frac{\delta T}{T} \nabla \cdot F^{\prime} \mathrm{d} v
$$

As the lower zone is unstable $I_{1}$ is negative; but $I_{2}$ is positive. The whole model will be unstable if $I_{1}+I_{2}<0$.

\section{Excitation of the Sorting Layer}

In the adiabatic approximation the eigenfunction can be obtained solving the set of equations:

$$
\begin{aligned}
& \frac{\partial \xi_{0}}{\partial \omega}=(\nabla \mu-1) \xi_{0}+\pi-\theta \\
& \frac{\partial \pi}{\partial \omega}=\nabla \mu \xi_{0}-\theta \\
& \left(\nabla_{T}-\nabla_{T a}\right) \xi_{0}+\left(1-\nabla \varrho_{a}\right) \pi-\theta=0
\end{aligned}
$$


We assume that in the layer the different gradients are constant. The second order differential equation giving $\xi_{0}$ is

$$
\ddot{\xi}_{0}+(2-\nabla \varrho) \dot{\xi}_{0}+(\dot{\lambda}-\nabla \varrho) \xi_{0}=0
$$

And the general solution can be written

$$
\xi_{0}=A \exp (-\omega)[1+\exp (\nabla \varrho \omega+\alpha)]
$$

We set the upper boundary of the layer at $\omega=0$ and assume $\xi_{0}=1$ i.e. $\alpha=0, A=\frac{1}{2}$. And the eigenfunction is

$$
\xi_{0}=\frac{1}{2} e^{-\omega}\left[1+e^{\nabla \varrho \omega}\right]
$$

In the quasi adiabatic approximation $\delta T / T=\theta-\xi \nabla_{T}$ using (26) becomes

$$
\delta T / T=\nabla_{T a}(\pi-\xi)
$$

and $\nabla \cdot F^{\prime}$ is computed from $(21)$.

The adiabatic expression for $\pi$ is derived from (24)

$$
\pi=-\frac{\nabla \varrho-\nabla \varrho_{a}}{2 \nabla \varrho_{a}} e^{-\omega}+\frac{1}{2} e^{(\nabla \varrho-1) \omega}
$$

Then

$$
\nabla \cdot F^{\prime}=\begin{gathered}
F \\
H_{p}
\end{gathered}\left\{\left(-a+b \begin{array}{c}
\nabla_{\varrho} \\
\nabla_{\varrho a}
\end{array}\right) e^{-\omega}+a(\nabla \varrho-1) e^{(\nabla \varrho-1) \omega}\right\}
$$

where $a$ and $b$ are expressed in terms of characteristic quantities of the layer

$$
\begin{aligned}
& a=-1-\kappa_{\mu} \nabla \mu-\kappa_{p}-\left(\kappa_{T}-4\right) \nabla_{T} \\
& b=-\frac{\nabla_{T q}}{\nabla_{T}}-\kappa_{p}-\left(\kappa_{T}-4\right) \nabla_{T q}
\end{aligned}
$$

$\chi_{\mu}, \chi_{T}, \chi_{p}$ being the logarithmic derivatives of $\chi$ with respect to $\mu, T$ and $p$. The contribution of this layer to the damping integral is:

$$
I_{1}=-\int_{\omega_{1}}^{0} \frac{\delta T}{T} \nabla \cdot F H_{p} \mathrm{~d} \omega
$$

Performing the integration one obtains

$$
I_{1}=F \nabla_{T a} \frac{\nabla \varrho}{2 \nabla \varrho_{a}}\left[g\left(\omega_{1}\right)-g(0)\right]
$$

where

$$
\omega_{1}=\log \underset{p_{0}}{p_{1}} \text { and } g(\omega)=\frac{1}{2}\left(-a+b \frac{\nabla \varrho}{\nabla \varrho_{a}}\right) e^{-2(\omega)}-\frac{a(\nabla \varrho-1)}{\nabla \varrho-2} e^{(\nabla \varrho-2) \omega}
$$




\section{Damping by the Outer Layers}

The boundary of a possible semi convective zone is situated in the very outerlayers $(\tau \simeq 0.2)$ so that it is impossible to keep the quasi adiabatic approximation in this region.

On the other hand, as the temperature relaxation time scale is very short one can assume that the perturbation has always the same temperature as the medium i.e. $\theta=0$. The eigenfunction can be computed from

$$
\begin{gathered}
\frac{\partial \xi_{0}}{\partial \omega}=-\xi_{0}+\pi \\
\frac{\partial \pi}{\partial \omega}=-{ }^{\sigma^{2} H_{p}} \xi_{0}
\end{gathered}
$$

As $\sigma^{2} H_{p} / g \ll 1$ the solution is

$$
\begin{aligned}
\zeta a & =K_{1} e^{-\omega}+K_{2} e^{-\left(\sigma^{2} H_{P} / g\right) \omega} \\
\pi & =K_{2} e^{-\left(\sigma^{2} H_{P} / g\right) \omega}
\end{aligned}
$$

Boundary conditions at $\omega=0$ fix the constants. They are

$$
\begin{aligned}
& \xi_{1}(0)=\xi_{2}(0) \\
& \pi_{1}(0)=\pi_{2}(0)
\end{aligned}
$$

where index 1 and 2 refer to the lower and upper zones respectively

$$
\begin{aligned}
& K_{1}+K_{2}=1 \\
& K_{2}=\frac{1}{2}-\frac{\nabla \varrho-\nabla \varrho_{a}}{2} \\
& K_{2}=\underset{2 \nabla \varrho_{a}}{\nabla} K_{2}=1-\begin{array}{c}
\nabla \varrho \\
2 \nabla \varrho_{a}
\end{array}
\end{aligned}
$$

$\nabla \cdot F^{\prime}$ is computed using (22) with $\theta=0$

$$
(\nabla \cdot F)^{\prime}=\frac{F \quad \partial \varphi}{H} \frac{\partial \omega}{\partial \omega}=-\frac{i \sigma p}{\nabla_{T a}}\left[\left(\nabla_{T}-\nabla_{T a}\right) \xi+(1-\nabla \varrho a) \pi\right]
$$

keeping only the first term in $e^{-\omega}$

$$
\nabla \cdot F=-\frac{i \sigma p}{\nabla_{T a}}\left(\nabla_{T}-\nabla_{T a}\right) K_{1} e^{-\omega}
$$

$\delta T / T$ is estimated from Equation (21) where $\nabla_{\mu}=0$ and $\theta=0$

$$
\partial \theta / \partial \omega=\left(1+\chi_{p}\right) \nabla_{T} \pi+\nabla_{T} \varphi
$$

This equation is the diffusing approximation of the radiative transfer equation. In the outer layers it can give only rough orders of magnitude. 
As $\nabla \cdot F^{\prime}$ is in quadrature with $\xi$ and $\pi$, we only need the part which is in quadrature with $\xi$ in $\delta T / T$ i.e.

$$
(\delta T / T)^{*}=\theta^{*} \text { with } \partial \theta^{*} / \partial \omega=\nabla_{T} \varphi
$$

Using the expression of $\partial \varphi / \partial \omega$ from Equation (22) and performing two successive integrations $\left(\varphi=\theta^{*}=0\right.$ at $\left.p=0\right)$ one gets

$$
\theta^{*}=\begin{gathered}
i \sigma \nabla_{T}-\nabla_{T a} \\
F \\
\nabla_{T a}
\end{gathered} \frac{\nabla_{T}}{(1-\nabla \varrho)^{2}} K_{1} p_{0} H_{p}
$$

and then the contribution of this region to the damping integral is

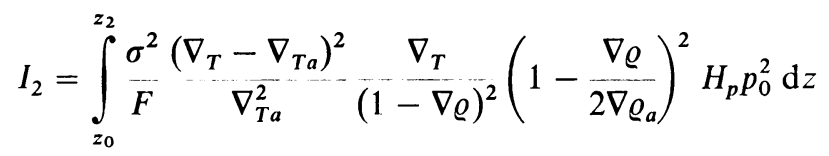

i.e.

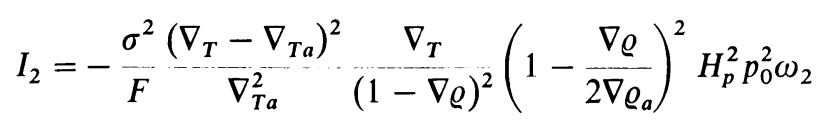

\section{Numerical Estimates for $I_{1}$ and $I_{2}$}

The typical model gives

$$
\begin{aligned}
F & =10^{11.1} \\
\sigma & \simeq 10^{-1} \\
H_{p} & \simeq 10^{4} \mathrm{~cm} \\
p_{0} & =10^{5.5} \mathrm{cgs}
\end{aligned}
$$

We will stop the integration in the outer atmosphere at $\omega_{2}=-3$. This boundary is completely arbitrary but one sees that $I_{2}$ is proportional to $\omega_{2}$ and so not very sensitive to the position of this limit. In the outer layer the mean values of the gradients are:

$$
\begin{aligned}
\nabla_{T} & \simeq 0.1 \\
\nabla_{T a} & \simeq 0.04
\end{aligned}
$$

So that $I_{2} \simeq 10^{7}$

In the region of varying molecular weight, we assume a Kramer's opacity law and the model gives the following mean values

$$
\begin{aligned}
\nabla \mu & =0.1 \\
\nabla_{T a} & =0.4 \\
\nabla \varrho & =0.8 \\
\omega_{1} & =1.3
\end{aligned}
$$

So that $g\left(\omega_{1}\right)-g(0)=-0.32$ and $I_{1} \simeq-10^{8}$ so that $I_{1}+I_{2}<0$. 


\section{Conclusion}

The result obtained here that the instability of the whole star could be triggered by the zone of varying molecular weight is only an indication that such a mechanism could work actually.

Very rough approximations are necessary to carry all along analytical expressions. A treatment including a better equation of transfer in the outer layers and a complete resolution of the differential system is needed to conclude. The region where $\nabla_{T a}<\nabla_{T}$ coincides with the region of second ionization of helium and we have omitted completely to take into account the variation of the degree of ionisation.

From the expressions obtained from $I_{1}$ and $I_{2}$ one sees that high order modes are more favorable to instability. If the effective temperature increases $H_{p}^{2} / F^{2}$ decreases and instability is favored but on the other hand the radiative gradient remains too small and the condition $\nabla_{T a}<\nabla_{T}$ is never satisfied. So that only a small interval in $T$ is favorable to that process.

It also implies that the zone of sorting is very close to the surface which means that there is very little hydrogen left.

\section{References}

Auré, J. L.: 1970, Astron. Astrophys., in press.

Chapman, S. and Cowling, T. G.: 1960, The Mathematical Theory of Non-Uniform Gases, Reprinted Dover, New York.

Gabriel, M.: 1969, Astron. Astrophys. 1, 321.

Kato, S.: 1966, PASJ 18, 374.

Ledoux, P. and Walraven, T.: 1958, Handbuch der Physik 51, Springer-Verlag.

Schatzman, E.: 1958, White Dwarfs, North-Holland Publ. Co., Amsterdam.

Vauclair, G.: 1970, to be published. 